\title{
1 Einleitung
}

Im Spannungsfeld zwischen globaler Finanz-, Rohstoff- und Nahrungsmittelpreiskrise sowie dem Klimawandel und seinen möglichen Auswirkungen beobachten Wissenschaftler*innen seit Mitte der 200oer Jahre eine zunehmende Nachfrage nach Land (Borras/Franco 2012). Zyklisch steigende Energieund Nahrungsmittelpreise, ein erhöhter Bedarf an Rohstoffen, Mineralien und Agrarkraftstoffen sowie die Notwendigkeit neuer Kapitalanlagemöglichkeiten haben Land zu einem gefragten Investitions- und Spekulationsobjekt gemacht (Akram-Lodhi 2012). Ausdruck für diese Entwicklung ist die $\mathrm{Zu-}$ nahme großflächiger Aneignungen von Land durch nationale, internationale und multinationale Akteure wie Unternehmen, Regierungen oder lokale Eliten (Dietz/Engels 2020). Typische Ziele dieser Landaneignungen sind neben der Spekulation die kommerzielle Nahrungsmittel-, Futtermittel- und Agrarkraftstoffproduktion, die Kommodifizierung der Senkenfunktion von Wäldern, Naturschutzmaßnahmen oder der Abbau von Mineralien und Rohstoffen (Borras et al. 2016; Cotula 2012; Edelman et al. 2013; Hall et al. 2015; Schoneveld/German 2014). Für landwirtschaftliche Zwecke allein belaufen sich die seit dem Jahr 2000 vertraglich abgesicherten Landaneignungen laut Nolte et al. (2016) weltweit auf eine Fläche von 26,7 Millionen Hektar. Sie konzentrieren sich vor allem in Afrika, Asien, Lateinamerika und Südosteuropa, wobei der afrikanische Kontinent mit 10 Millionen Hektar flächenmäßig am stärksten von dieser Entwicklung betroffen ist (ebd.: 16). Dort eignen sich Investoren insbesondere gewohnheitsrechtlich verwaltetes und kollektiv genutztes Land an (Dell'Angelo et al. 2017). In diesem Buch bezeichne ich das gegenwärtige Phänomen der flächen- und zahlenmäßig zunehmenden Aneignungen von Land als land grabbing. Land grabbing verstehe ich in Anlehnung an Borras et al. (Borras/Kay/et al. 2012: 405) als Aneignung der Kontrolle über relativ große Flächen an Land und anderen Ressourcen, die mit einer intendierten Inwertsetzung einhergeht und eine Verlagerung weg von der kleinbäuerli- 
chen, subsistenzorientierten Landwirtschaft hin zu einer agrarindustriellen, marktorientierten Landwirtschaft mit sich bringt. Mit der Wahl dieses Terminus grenze ich mich von synonym verwendeten Bezeichnungen wie Investitionen in oder Transaktionen von Land $a b$ und rücke insbesondere die Veränderung von Kontrollverhältnissen in den Fokus.

Land grabbing geht in vielen Fällen mit tiefgreifenden Veränderungen der Landnutzung und der Landbesitzverhältnisse einher. In diesem Zusammenhang entstehen zunehmend Konflikte, die auf unterschiedlichen Ebenen und zwischen unterschiedlichen Akteuren verlaufen (Borras/Franco 2013; Engels/Dietz 2017; Peters 2013a). Diese Konflikte treten typischerweise in drei Akteurskonstellationen auf: zwischen Landnutzer*innen und Unternehmen, Landnutzer"innen und staatlichen Akteuren sowie zwischen unterschiedlichen Gruppen von Landnutzer*innen (Borras/Franco 2013). Zentrale Themen in diesen Konflikten sind die Ausbeutung, Vertreibung und Enteignung von Landnutzer"innen oder Landbewohner*innen durch land grabbing, antagonistische Interessen sozial differenzierter Gruppen bezüglich der geplanten Vorhaben, die Konkurrenz von unterschiedlichen Landnutzer"innengruppen um verbleibende Nutzflächen sowie die Zerstörung und Verschmutzung der Umwelt (Borras/Franco 2013). Das verbindende Element in all diesen Konflikten ist die Aushandlung von Zugang zu Land, wobei Zugang zu Land bedeutet, dass Akteure einen Nutzen aus dem Land ziehen können (Blaikie 1985; Ribot/Peluso 2003). Aus dieser Perspektive verlaufen Konflikte zwischen Akteuren, die Zugang zu Land kontrollieren, denjenigen die sich Zugang neuaneignen, und denjenigen, die Zugang über Dritte erlangen, um ihren Lebensunterhalt zu bestreiten. Je nach Ausgestaltung des Zugangs zu Land sind in den Konflikten unterschiedliche Akteure involviert.

In vielen Ländern Subsahara-Afrikas ist Zugang zu Land durch rechtsund normpluralistische Landverwaltungssysteme bestimmt, in denen traditionelle, informelle und staatliche Institutionen und Gesetze zum Tragen kommen. Diese können parallel zueinander existieren, komplementär sein, sich überlagern oder in Konkurrenz zueinander stehen (Bierschenk 2010; Lund 2006; Olivier de Sardan 2011; Ubink 2008b). Rechts- und normpluralistische Strukturen in der Landverwaltung sind historisch spezifisch, dynamisch und nicht per se konfliktiv. Wenn in diesen Kontexten jedoch Konflikte um Land auftreten, stellen die unterschiedlichen rechtlichen und normativen Grundlagen einen wichtigen Ausgangspunkt für die Analyse dieser Konflikte dar (Bierschenk/Olivier de Sardan 1997; Boamah 2014a; Lund 1998, 2006). 
Schätzungsweise 70 Prozent aller land- und viehwirtschaftlichen Nutzflächen in Subsahara-Afrika unterliegen der Verwaltung im Rahmen von Gewohnheitsrechten. Diese haben, im Gegensatz zu staatlichem Recht, ihren Ursprung nicht in der staatlichen Politik. Dennoch sind Gewohnheitsrechte in vielen Ländern gesetzlich anerkannt oder zumindest, mangels Alternativen akzeptiert. Die gewohnheitsrechtliche Verwaltung von Land erfolgt durch traditionelle Institutionen. Als traditionelle Institutionen bezeichne ich historisch und sozial generierte und verinnerlichte Verhaltenserwartungen, und -weisen, die sich als grundlegende Struktur durch das jeweilige System ziehen und soziale Interaktion regeln. Sie ermöglichen oder beschränken das Handeln sozialer Akteure. Typischerweise sind diese Institutionen informell. Regeln und Verfahrensweisen sind nicht verschriftlicht und treten nicht über »offizielle«, staatliche Wege in Kraft, sondern erlangen ihre Geltung durch Autolizenzierung und hängen in ihrem Fortbestand von ihrer faktischen Anerkennung ab (Helmke/Levitsky 2004: 727). »Traditionell« bezieht sich hier auf Institutionen die im Gegensatz zu den Institutionen des postkolonialen Staates ein gewisses kulturelles Erbe abbilden, das aus der Gesellschaft selbst hervorgegangen ist (Englebert 2002a: 366), unabhängig davon, ob dieses Erbe wahr, erfunden oder ausgedacht ist (vgl. Ranger 1983). »Traditionell« bedeutet somit nicht, praktizierte Bräuche stammten aus einer unberührten präkolonialen Vergangenheit, seien zeitlos, unveränderbar und anachronistisch. Ich schließe mich dieser Auffassung an und grenze mich von einem Verständnis von Tradition als mangelnde Modernität oder als Relikt aus der Vergangenheit, das es zu überwinden gilt, ab. Vielmehr gehe ich davon aus, dass sich Tradition im Zusammenhang mit gesellschaftlichem, wirtschaftlichem und politischen Wandel entwickelt und verändert. Das erklärt auch die unterschiedliche Ausgestaltung des Gewohnheitsrechts in seiner zeitlichen und räumlichen Dimension. Gewohnheitsrechte unterscheiden sich von Dorf zu Dorf, von Region zu Region und von Staat zu Staat.

In traditionellen Herrschaftssystemen liegt die Verwaltung von Land maßgeblich in der Verantwortung traditioneller Autoritäten, die sich ebenso wie die traditionellen Systeme selbst - durch eine große Vielfalt auszeichnen (von Trotha 1996: 80). Diese Vielfalt bezieht sich unter anderem auf das Verhältnis traditioneller Autoritäten zu staatlichen Akteuren, auf ihre Legitimationsgrundlage, ihre Ressourcen, Handlungsspielräume und ihre Zuständigkeitsbereiche (Ubink 2008c). Unterschiedliche Bezeichnungen für traditionelle Autoritäten tragen dieser Vielfalt Rechnung. Im englischsprachigen Raum finden häufig die Termini chiefs und traditional authorities 
Verwendung, es existieren jedoch auch Bezeichnungen wie clan head, family head, headman, queen mother, spiritual leader oder earth priest. Das Pendant dazu im französischsprachigen Raum sind die chefs de village, chefs de terre und chef coutumiers. Da es im Deutschen keine adäquate Übersetzung für diese Begriffe gibt, verwende ich im Folgenden die Bezeichnungen chief und traditionelle Autorität synonym. Sie können sich sowohl auf weibliche als auch auf männliche Titelträger*innen beziehen. Unabhängig von ihrer Bezeichnung ist allen traditionellen Autoritäten gemein, dass ihre Anerkennung, Legitimität und Macht auf ihrer Zugehörigkeit zu einer ortsbezogenen Gemeinschaft beruhen, über die sie unter Berücksichtigung gewohnheitsrechtlicher Bräuche herrschen (Baldwin 2016: 21).

In vielen Ländern - wie in Ghana, Mali, Zimbabwe, Malawi, oder Ugan$\mathrm{da}$ - sind traditionelle Autoritäten die zentralen Entscheidungsträger*innen bezüglich der Vergabe von gewohnheitsrechtlich verwaltetem Land an kleinbäuerliche Nutzer*innen (Logan 2013). Lange Zeit waren Gewohnheitsrechte von staatlicher Seite aus eher geduldet als tatsächlich anerkannt. Seit den 1990er Jahren gibt es jedoch eine Tendenz zur staatlichen Anerkennung dieser Rechte (Knierzinger 2011; Ubink 2008c). Gleichzeitig hat sich durch Landrechtsreformen, Dezentralisierungs- und Strukturanpassungsprogramme sowie die Liberalisierung der Marktwirtschaft seit den frühen 1990er Jahren auch die rechtliche Ausgestaltung und Strukturierung des Landsektors in Ländern des Globalen Südens ${ }^{1}$ verändert. In diesem Zusammenhang haben traditionelle Autoritäten ebenfalls an Einfluss in der Landverwaltung gewonnen (Ubink 2008c). In jüngster Zeit treten sie im Kontext von land grabbing als zentrale Akteure in der großflächigen Vergabe von Land an Investoren in Erscheinung (Ahmed et al. 2018; Capps 2016, 2018). Den Begriff der großflächigen Vergabe von Land nutze ich synonym zu land grabbing, hebe dadurch aber die Beteiligung traditioneller Autoritäten hervor. Die Tatsache, dass land grabbing oftmals mit Konflikten um den Zugang zu Land einhergeht, macht traditionelle Autoritäten für die Analyse von Konflikten um Zugang

1 Die Bezeichnungen Globaler Süden und Globaler Norden verweisen nicht auf die geographische Lage von Staaten, sondern auf Ungleichheitsverhältnisse in den weltpolitischen und wirtschaftlichen Positionen der Länder sowie auf unterschiedliche Erfahrungen mit Kolonialismus und Ausbeutung. Der Begriff Globaler Süden bezieht sich nicht auf einzelne Nationalstaaten, sondern auf die Gesamtheit der marginalisierten Bevölkerung (de Sousa Santos 2016: 19). Der Globale Norden umfasst im Gegenzug all diejenigen, die im globalen Machtgefüge eine privilegierte Position einnehmen (glokal 2013: 8). 
in diesem Zusammenhang zu Schlüsselfiguren. Welche Rolle traditionelle Autoritäten in diesen Konflikten spielen, ist bislang jedoch kaum erforscht. In diesem Buch gehe ich folgender Forschungsfrage nach: Welcher Zusammenhang besteht zwischen dem Handeln traditioneller Autoritäten und Konflikten um Zugang zu Land im Kontext von land grabbing? Im Zentrum des Erkenntnisinteresses stehen drei Fragen: (1) Wie verändert sich Kontrolle über den Zugang zu Land im Kontext von land grabbing? (2) Wie verändern sich in diesem Zusammenhang traditionelle Herrschaftssysteme? Und (3) wie wirkt sich das Handeln traditioneller Autoritäten auf die Entstehung und den Verlauf von Konflikten um Zugang zu Land aus?

\subsection{Problemstellung}

Ausgangspunkt meiner Forschung ist die Beobachtung, dass in rechts- und normpluralistischen Landverwaltungssystemen Subsahara-Afrikas traditionelle Autoritäten im Kontext von land grabbing zunehmend als Verhandlungspartner"innen von Investoren für die Vergabe von Land verantwortlich sind (Boamah 2014a: 407; Lanz et al. 2018; Yaro/Tsikata 2014). Aktuelle Studien belegen, dass im Zusammenhang mit land grabbing oftmals Konflikte auftreten und dass traditionelle Autoritäten aufgrund ihrer Zuständigkeit für die Vergabe von Land zentrale Akteure in diesen Konflikten sind, in denen sie gleichzeitig auch als Mediator*innen agieren (Campion/Acheampong 2014). Darüber hinaus gibt die bestehende Literatur zu Konflikten, die im Zusammenhang mit land grabbing auftreten, wenig Aufschluss darüber, welche Rolle traditionelle Autoritäten in diesen Konflikten spielen, wie sich traditionelle Herrschaftssysteme in Verbindung mit land grabbing verändern und warum in diesem Zusammenhang Konflikte auftreten. In Bezug auf Akteurskonstellationen konzentrieren sich Analysen zu land grabbing und Konflikten bislang vornehmlich auf die Rolle des Staates (Busscher et al. 2019; Hall et al. 2015; Wolford et al. 2013), von Unternehmen (Oya 2013; Scoones et al. 2013) und sozialen Bewegungen (Engels 2019; Prause 2019). Auch wissenschaftliche Arbeiten zu traditioneller Herrschaft geben keinen Aufschluss zu traditionellen Autoritäten als Konfliktakteure, sondern interessieren sich insbesondere für ihre Rolle als Intermediäre, Entwicklungsagent*innen (Bierschenk et al. 2000; Kleist 2011; Knierzinger 2011) und Mediator"innen (Rukuni et al. 2015; Santschi 2014; Tubiana et al. 2012). Die aktuelle Forschung gibt keinen Aufschluss darüber, wie sich traditionelle Herrschaft im Kontext von land grabbing ver- 
ändert, inwiefern sich mögliche Veränderungen auf den Zugang zu Land von Landnutzer*innen auswirken und warum in diesem Kontext Konflikte entstehen. Konflikte verstehe ich als soziale Beziehungen zwischen mindestens zwei individuellen oder kollektiven Akteuren gekennzeichnet durch von ihnen als widersprüchlich wahrgenommene Interessen, Ziele oder Bedürfnisse (Bonacker/Imbusch 2006). Traditionelle Autoritäten gelten als Repräsentant*innen ihrer Gemeinschaft und ihre Aufgabe ist es, die Interessen dieser Gemeinschaft zu vertreten. Wie erklären sich also zunehmende Widersprüche und damit einhergehende Konflikte in traditionellen Landverwaltungssystemen im Zusammenhang mit land grabbing, und welchen Einfluss haben traditionelle Autoritäten auf die Entstehung und den Verlauf dieser Konflikte?

Ziel meiner Forschung ist, es empirisch begründetes Wissen zur Erklärung von Konflikten um Zugang zu Land, die in traditionellen Landverwaltungssystemen im Zusammenhang mit land grabbing auftreten, zu generieren. Meine erste Annahme diesbezüglich ist, dass das Handeln traditioneller Autoritäten als Schlüsselfiguren in der Vergabe von Land zentral für die Entstehung und den Verlauf dieser Konflikte ist. Der Gegenstand meiner Analyse ist daher das Handeln traditioneller Autoritäten. Diesbezüglich beziehe ich mich auf relationale Erklärungsansätze von Konflikten. Diesen zufolge können Konflikte nicht allein über das Handeln einer Person erklärt werden, sondern nur unter Berücksichtigung der Interaktion mit anderen Akteuren. Das bedeutet für meine Analyse, dass ich das Handeln traditioneller Autoritäten hinsichtlich der Interaktion mit anderen Akteuren, die in die Landvergabe einbezogen oder von ihr betroffen sind, untersuche. Meine zweite Annahme ist, dass die Ausgestaltung traditioneller Herrschaftssysteme das Handeln traditioneller Autoritäten beeinflusst, was sich wiederum auf Konflikte auswirken kann. In meinem Forschungsdesign habe ich mich daher für eine kollektive Fallstudie entschieden. Eine kollektive Fallstudie bezeichnet die Ausweitung einer Einzelfallstudie auf zwei oder mehrere Fälle (Stake 2003: 138). Die Erforschung von zwei Fällen ermöglicht mir, Unterschiede und Parallelen im Handeln traditioneller Autoritäten in unterschiedlichen Herrschaftssystemen zu untersuchen und verallgemeinerbare Hypothesen zu generieren.

Hinsichtlich der bestehenden Literatur beziehe ich mich auf Studien zu (1) Konflikten im Zusammenhang mit land grabbing, (2) Land, sozialer Zugehörigkeit und Konflikten und (3) traditionellen Autoritäten als Intermediäre. Der Bezug auf diese drei Forschungsfelder ist aus folgenden Gründen für die Bearbeitung meiner Fragestellung sinnvoll. Beiträge zu Konflikten im Kontext von land grabbing liefern Anhaltspunkte dazu, warum diese Konflikte entste- 
hen, welche übergeordneten Fragen Akteure in diesen Konflikten verhandeln und welche Akteure in diesen Konflikten typischerweise handeln. Die Forschung zu Land, sozialer Zugehörigkeit und Konflikten rückt die Aushandlung von Zugang zu Land in traditionellen Landverwaltungssystemen in den Fokus. Beiträge zu diesem Thema widmen sich insbesondere der Bedeutung sozialer Zugehörigkeit und Differenzierung hinsichtlich der Aushandlung von Zugang zu Land in traditionellen Landverwaltungssystemen und geben Aufschluss darüber, welche Wechselwirkung dort zwischen Machtverhältnissen und der Kontrolle des Zugangs zu Land besteht. Für meine Forschung, in der traditionelle Landverwaltungssysteme den Kontext bilden, sind diese Beiträge eine sinnvolle Ergänzung der Literatur zu land grabbing und Konflikten. Das dritte Literaturfeld zu traditionellen Autoritäten als Intermediäre gibt Aufschluss über die geschichtliche Entwicklung der Rolle traditioneller Autoritäten im Spannungsfeld gesellschaftlichen, politischen und wirtschaftlichen Wandels und ermöglicht ein differenziertes Verständnis der möglichen Herausforderungen und Widersprüche, die sich aus dem Handeln traditioneller Autoritäten ergeben können.

In der empirischen Analyse identifiziere ich typische Handlungsstrategien traditioneller Autoritäten in der Landvergabe an Investoren in unterschiedlichen traditionellen Herrschaftssystemen. Ich untersuche, inwiefern sich diese Handlungsstrategien auf den Zugang zu Land von unterschiedlichen Landnutzer*innen sowie auf die Entstehung und den Verlauf von Konflikten auswirken. Die gewonnenen Erkenntnisse bilden die Grundlage für die Entwicklung von Thesen, die der Erweiterung theoretischer Zugänge zu Konflikten um Zugang und ihrer sozio-politischen Vermittlung in traditionellen Landverwaltungssystemen dienen können.

\subsection{Forschungskontext und Forschungsdesign}

Während der Kolonialzeit galten traditionelle Autoritäten als der verlängerte Arm der Kolonialverwaltung. Grund dafür war ihre der administrative chiefs, die sie in vielen Ländern Subsahara-Afrikas innehatten (Mamdani 1996: 52-61; von Trotha 1996). Entsprechend niedrig war ihre Beliebtheit bei den sich neu formierenden, nach Unabhängigkeit strebenden nationalen Regierungen. Das Bild traditioneller Autoritäten als Kollaborateur*innen der Kolonialmächte hatte auch nach der Unabhängigkeit der Kolonialstaaten Bestand. Es gab allerdings Unterschiede in der Haltung der postkolonialen Regierungen 
gegenüber traditionellen Autoritäten. Viele standen traditionellen Autoritäten aufgrund iherer geringen Beteiligung an den Unabhängigkeitskämpfen weiterhin kritisch gegenüber (Baldwin 2016: 32). In einer Ära der Demokratisierung galten traditionelle Autoritäten zudem als antidemokratische lokale Despoten (Baldwin 2016: 3). Postkoloniale Regierungen nahmen sie als Konkurrent*innen oder als Bedrohung ihrer Macht wahr. Über unterschiedliche Wege versuchten sie den Einfluss traditioneller Autoritäten in der Lokalverwaltung und nationalen Politik zu beschränken. Typische Maßnahmen waren die formelle Abschaffung traditioneller Herrschaftssysteme, die Beschränkung der Handlungsmöglichkeiten traditioneller Autoritäten in zentralen Bereichen wie Bildung, Gesundheit und Landverwaltung oder die Ignoranz traditioneller Herrschaft. Letztere konnte ebenso zu einer Wiederbelebung wie auch zum Bedeutungsverlust traditioneller Herrschaft führen (Ubink 2008c: 10; von Trotha 2000: 257). Insgesamt verschwand sie jedoch nicht. Im Gegenteil: Mehr als 50 Jahre nach den ersten Unabhängigkeitserklärungen wird deutlich, dass die meisten Versuche, traditionelle Herrschaft durch staatliche Institutionen $\mathrm{zu}$ ersetzen, scheiterten. Zahlreiche Wissenschaftler*innen zeigen in ihren Forschungen, dass traditionelle Autoritäten eine erstaunliche Anpassungsfähigkeit an soziopolitischen Wandel unter Beweis stellen und weiterhin in der Lage sind, Einfluss in zentralen Bereichen wie der Lokalverwaltung, Konfliktschlichtung oder Landverwaltung auszuüben (Baldwin 2016; Berry 1992; van Rouveroy van Nieuwaal 1996: 45). Seit den 1990er Jahren interessieren sich afrikanische Regierungen und internationale Geberorganisationen wieder zunehmend für traditionelle Autoritäten (Ubink 2008c: 11) und Wissenschaftler*innen sprechen von einer »Wiederbelebung« traditioneller Herrschaft (Bräuchler/Widlok 2007; Buur/Kyed 2007; Englebert 2002b). In Ländern wie Uganda, Mosambik, Südafrika und Ghana - in denen traditionelle Herrschaft zwar nach der Unabhängigkeit nicht formell abgeschafft wurde, jedoch deutliche Restriktionen erfuhr - ging diese Wiederbelebung sogar mit einer verfassungsrechtlichen Formalisierung des chieftaincy-Systems einher (Ubink 2008c).

Der Bedeutungsgewinn traditioneller Herrschaft steht auch im Zusammenhang mit der zunehmenden Kritik von Geberorganisationen und der internationalen Entwicklungspolitik an der institutionellen Qualität in vielen afrikanischen Staaten, insbesondere auf lokaler Ebene. Die schwachen Kapazitäten und die mangelnde Leistungsfähigkeit lokaler staatlicher Institutionen machte etwa die Weltbank für die vermeintlich schlechte wirtschaftliche Entwicklung verantwortlich (Brautigam 1992; Hydén/Bratton 1992; World 
Bank 1989, 1992, 1994). Die Kooperation mit traditionellen Autoritäten stellte für die Weltbank einen möglichen Lösungsansatz für dieses Problem dar (World Bank 2002). Die gezielte Einbindung traditioneller Autoritäten in die Lokalpolitik, zum Beispiel im Rahmen von Dezentralisierungsbestrebungen, sollte die Regierungsführung auf lokaler Ebene stärken und fehlende staatliche Kapazitäten ausgleichen. Hinzu kam, dass chiefs sich bei mangelnder Einbeziehung in vielen Fällen der Umsetzung staatlicher Politik auflokaler Ebene widersetzten und angestrebte Maßnahmen dadurch scheiterten (Stacey 2015).

Die Kooperation mit traditionellen Autoritäten steht in Einklang mit einer dem Selbstanspruch nach partizipatorischen Entwicklungspolitik (OECD/DAC 1996: 9). Vor diesem Hintergrund gewannen traditionelle Autoritäten in Subsahara-Afrika an Prominenz. In der entwicklungspolitischen Praxis erlangten sie als Intermediäre an Bedeutung. Wissenschaftliche Studien verhandeln diese neue Rolle unter den Bezeichnungen Entwicklungspartner*innen (agents of development) und Entwicklungsvermittler*innen (development broker) (Baldwin 2016: 69ff.; Grischow 2008; Knierzinger 2011; Yaro/Tsikata 2014). Als Intermediäre zwischen internationalen Geber*innen und lokalen Empfänger*innen sind sie für die Vernetzung von Entwicklungsakteuren und der lokalen Gemeinschaft, eine bessere sozio-kulturelle Verständigung, die Verwaltung von Projektgeldern, die Mobilisierung von Arbeitskräften und die Mediation in Konflikten zuständig (Knierzinger 2011: 28f.; Ubink 2008a: 141ff.). Darüber hinaus treten traditionelle Autoritäten in zentralen Bereichen wie dem Bildungs- oder Gesundheitssektor, in denen die Kapazitäten staatlicher Institutionen schwach sind, als Dienstleister*innen auf (Ubink 2008c: 14). Durch diese Tätigkeit steigen ihr Ansehen und ihre Legitimität innerhalb der Gemeinschaft, was mit der einer Stärkung ihrer gesellschaftlichen Stellung und der Ausweitung ihrer Macht einhergeht. Die Ausweitung ihrer Macht wiederum ermöglicht ihnen, Entscheidungen zu treffen, ohne ihrer Gemeinschaft oder staatlichen Akteuren gegenüber Rechenschaft ablegen $\mathrm{zu}$ müssen. So sind sie in der Lage, ihre Interessen durchzusetzen, auch wenn diese im Widerspruch zu den Interessen der Gemeinschaft stehen. Ein Beispiel dafür ist die Selbstbereicherung einiger chiefs an gemeinschaftlich genutzten Ressourcen wie Land (Ubink 2008c: 27).

Hinsichtlich der zentralen Bedeutung traditioneller Autoritäten in der Vergabe von Land an Investoren ist die Erkenntnis, dass traditionelle Autoritäten sich vermehrt selbst an gemeinschaftlich genutzten Ressourcen bereichern, von besonderer Relevanz. Sie lenkt den Blick auf die widersprüchlichen Rollen traditioneller Autoritäten. Sie sind Repräsentant*innen der Gemein- 
schaft und zuständig für Dienstleistungen, wie die Vergabe von Land oder den Ausbau des Gesundheits- und Bildungssektors. Gleichzeitig sind sie auch Akteure mit eigenen Interessen, die teilweise im Widerspruch zu den Interessen ihrer Gemeinschaft stehen. Das kann ein Ausgangspunkt für Konflikte sein.

Um untersuchen zu können, welcher Zusammenhang zwischen dem Handeln traditioneller Autoritäten und Konflikten um Zugang im Zusammenhang mit land grabbing besteht, entwickle ich einen Analyserahmen, der ein theoretisches Verständnis von Zugang $\mathrm{zu}$ Land in traditionellen Landverwaltungssystemen mit einem auf die empirische Analyse ausgerichteten mehrdimensionalen Machtkonzept verbindet. In traditionellen Landverwaltungssystemen erlangen Landnutzer*innen Zugang zu Land nicht ausschließlich über Besitz, sondern auch über eine Vielzahl weiterer rechtebasierter, relationaler und struktureller Mechanismen, zu denen verschiedene traditionelle Rechte, individuelle Vereinbarungen und persönliche Beziehungen zählen. Aufgrund meines Verständnisses von Konflikten im Zusammenhang mit land grabbing als Konflikte um Zugang zu Land gehe ich davon aus, dass die Frage, über welche Mechanismen Zugang zu Land erlangt wird, wichtig für die Erklärung dieser Konflikte ist. Ein theoretisches Verständnis von Zugang zu Land in traditionellen Landverwaltungssystemen soll ermöglichen zu erfassen, über welche Mechanismen Landnutzer*innen Zugang zu Land erlangen und inwiefern sich ihre Möglichkeiten auf diese Mechanismen zurückzugreifen im Kontext der großflächigen Landvergabe verändern. Mein theoretisches Verständnis von Zugang zu Land in traditionellen Landverwaltungssystemen entwickle ich auf der Grundlage der Theory of Access im Anschluss an Jesse Ribot und Nancy Peluso (Ribot/Peluso 2003). Diese erweitere ich durch Erkenntnisse aus der Forschung zu Zugang zu Land in traditionellen Landverwaltungssystemen Subsahara-Afrikas. Über die Analyse der Veränderung des Zugangs zu Land arbeite ich die zentralen Widersprüche heraus, die sich durch die großflächige Landvergabe ergeben und sich in Konflikten um Zugang manifestieren. Um zu verstehen, in welchem Zusammenhang das Handeln traditioneller Autoritäten mit Konflikten um Zugang steht, fehlt ein erklärendes Element. Dieses erklärende Element soll ermöglichen zu analysieren, wie es Konfliktakteuren - und insbesondere den für die Landvergabe zuständigen traditionellen Autoritäten - gelingt ihren Zugang zu Land und den Zugang Dritter im Kontext der großflächigen Landvergabe aufrechtzuerhalten oder zu kontrollieren. Viele Autor*innen betonen, dass die Fähigkeit Kontrolle über den Zugang zu Land auszuüben 
eine Frage von Machtverhältnissen ist (Bryant/Bailey 1997; Peluso/Lund 2011; Peluso/Ribot 2020). In welcher Form Akteure Macht ausüben, um Zugang $\mathrm{zu}$ Land zu kontrollieren bleibt jedoch unklar. Daher kombiniere ich in meinem Analyserahmen das theoretische Verständnis von Zugang zu Land in traditionellen Landverwaltungssystemen mit Steven Lukes' Konzept der drei Dimensionen von Macht (Lukes 2005). Dieses Konzept berücksichtigt Macht auf unterschiedlichen Ebenen: auf der Ebene (individueller) Entscheidungen, der Ebene institutioneller Verfahrensweisen sowie der Ebene gesellschaftlicher Kräfteverhältnisse. Das Konzept bietet sich für meine Forschung an, da es durch die Berücksichtigung unterschiedlicher Formen von Macht eine Offenheit gegenüber empirischen Phänomenen erlaubt. Damit eignet es sich für explorative Forschungsdesigns wie das meiner Studie, die darauf abzielen, neue Erkenntnisse zu bislang wenig erforschten Phänomenen zu gewinnen. Mithilfe dieses Konzepts untersuche ich, inwiefern traditionelle Autoritäten im Rahmen der Landvergabe Macht ausüben, um den Zugang $\mathrm{zu}$ Land kontrollieren zu können. Über die Untersuchung identifiziere ich typische Handlungsstrategien, über die traditionelle Autoritäten Zugang $\mathrm{zu}$ Land kontrollieren, und zeige, wie sich traditionelle Herrschaftssysteme dadurch im Kontext von land grabbing verändern. Anschließend analysiere ich, inwiefern sich diese Handlungsstrategien auf die Entstehung und den Verlauf von Konflikten auswirken.

\subsection{Fallauswahl}

In der empirischen Forschung konzentriere ich mich auf Ghana. Folgende vier Faktoren waren für die Auswahl dieses Fallstudienlands ausschlaggebend: erstens die Relevanz traditioneller Autoritäten in den Bereichen Landverwaltung und Konfliktmanagement, die in Konflikten um Zugang zu Land im Zusammenhang mit land grabbing zu der zuvor beschriebenen widersprüchlichen Position traditioneller Autoritäten führt; zweitens die unterschiedliche Ausgestaltung traditioneller Herrschaft innerhalb eines Länderkontexts; drittens das Ausmaß von land grabbing und viertens die Existenz zahlreicher Konflikte, die im Zusammenhang mit land grabbing auftraten, in denen traditionelle Autoritäten zu den Konfliktakteuren gehören. Die letzten beiden Faktoren sind eine notwendige Voraussetzung für die Identifizierung geeigneter Fälle. 
In Bezug auf die Verknüpfung von Landvergabe und Konfliktmanagement ist der Einfluss traditioneller Autoritäten in Ghana besonders groß. Die bis heute starke Stellung traditioneller Autoritäten in Ghana lässt sich durch unterschiedliche Faktoren erklären. Ghana zählt, neben Mosambik und Südafrika, zu den Ländern, in denen die post-kolonialen Regierungen die Stellung traditioneller Autoritäten ab den frühen 1980er Jahren gestärkt und formalisiert haben (Ubink 2008c: 11). Seit 1992 sind traditionelle Autoritäten und das chieftaincy-System in Ghana formal durch die Verfassung anerkannt (vgl. Kasanga/Kotey 2001). Zu Beginn der 2000er Jahre konnten traditionelle Autoritäten in Ghana die Weltbank und andere Geberorganisationen davon überzeugen, als Intermediäre zwischen lokaler und (inter)nationaler Ebene eine wichtige Rolle in der Umsetzung von Entwicklungsprojekten und Verwaltungsreformen einnehmen zu können. Für andere afrikanische Staaten mit starken traditionellen Herrschaftssystemen hatten sie eine Vorbildfunktion und trugen zur Wiederbelebung traditioneller Herrschaft in SubsaharaAfrika von internationaler Seite aus bei (Berry 2013: 38; World Bank 2002).

Traditionelle Herrschaft unterscheidet sich zwischen den Regionen Ghanas. Unterschiede in der traditionellen Herrschaft sind auf die unterschiedliche Einbeziehung traditioneller Autoritäten während der Kolonialzeit zurückzuführen. Infolgedessen ist traditionelle Herrschaft in der Ashanti Region stark hierarchisch organisiert, mit besonders mächtigen chiefs an der Spitze. In anderen Regionen ist traditionelle Herrschaft stärker durch Clanund Familien-Strukturen bestimmt oder eng mit spirituellen Aufgaben verknüpft. In meiner Forschung gehe ich davon aus, dass die unterschiedliche Ausgestaltung traditioneller Herrschaft ein möglicher Ansatzpunkt für die sozio-politische Vermittlung von Konflikten, die im Zusammenhang mit land grabbing auftreten, ist. Neben den bisher erläuterten relevanten Faktoren für die Fallauswahl eignet sich Ghana besonders als Fallstudienland, da es sich seit Beginn der $2000 e r$ Jahre zu einem beliebten Zielland für die Umsetzung großflächiger inter- und multinationaler agrarindustrieller Projekte entwickelt hat (Schoneveld/German 2014). Die Land Matrix verzeichnet für Ghana aktuell 100 Fälle von land grabbing durch inter- und multinationale Investoren (The Land Matrix 2020). In ihrer Erhebung berücksichtigt die Land Matrix Flächen mit einer Größe von 200 Hektar aufwärts (Nolte et al. 2016: 3). Damit liegt Ghana im Vergleich zu anderen Ländern Subsahara-Afrikas zahlenmäßig nur hinter Mosambik, Äthiopien und Tansania. Wie auch in anderen Ländern sind traditionelle Autoritäten in Ghana seit Beginn der 200oer Jahre zu wichtigen Verhandlungspartner*innen in der großflächigen Verga- 
be von Land an Investoren geworden (Cotula et al. 2014). Aufgrund der verfassungsrechtlichen Anerkennung traditioneller Herrschaft und der uneingeschränkten Zuständigkeit traditioneller Autoritäten in der Landverwaltung haben sie relativ freie Hand in den Verhandlungen mit Investoren. Zugleich sind sie aber auch dafür zuständig, die Interessen ihrer Gemeinschaft zu vertreten. Ihnen obliegt es, die Zugangsrechte der Landnutzer*innen zu schützen, im Fall des Verlusts von Land angemessene Entschädigungszahlungen durchzusetzen, Kleinbäuerinnen und -bauern in die Verhandlungen miteinzubeziehen und ihnen die Möglichkeit des Widerspruchs oder der Überprüfung von Vereinbarungen zu geben (Elhadary/Obeng-Odoom 2012; Vermeulen/Cotula 2010: 907). Dieser Aufgabe kommen viele von ihnen nicht nach, sondern handeln aus Eigeninteresse. Wie auch in anderen Ländern treten im Verlauf der Verhandlungen und während der Implementierung der Projekte in Ghana häufig Konflikte auf: Konflikte zwischen unterschiedlichen Gruppen von Landnutzer*innen, Konflikte, die durch die unrechtmäßige Vergabe von Land oder die Intransparenz der Verhandlungen entstehen, und Konflikte zwischen unterschiedlichen Nutzer*innengruppen wie Kleinbäuerinnen und -bauern und mobilen Viehzüchter*innen (Tsikata/Yaro 2011). Aufgrund ihrer zentralen, aber auch ambivalenten Rolle in der Vergabe großer Flächen an Land zählen traditionelle Autoritäten in Ghana zunehmend zu den Konfliktakteuren. Welche Rolle sie in diesen Konflikten einnehmen, ist in der Forschung bislang kaum untersucht worden (Boamah 2014a: 407).

Ich analysiere das Handeln traditioneller Autoritäten in Konflikten um großflächige Landtransaktionen in Ghana anhand eines Vergleichs von zwei Fällen: die Konflikte um das ScanFarm-Projekt in Ashanti und das BioFuel Africa-Projekt in der Northern Region. Beide Fälle sind Konflikte um agrarindustrielle Vorhaben, die ursprünglich den Anbau von Jatropha (bot. jatropha curcas) anstrebten. Jatropha ist eine strauchige Pflanze, deren Samen stark ölhaltig sind. Das aus ihnen gewonnene Öl dient der Produktion von Agrarkraftstoffen. Der großflächige Anbau von Energiepflanzen - und insbesondere von Jatropha - mit dem Ziel der Agrarkraftstoffproduktion hat sich vielerorts als besonders konfliktreich herausgestellt. Ab Mitte der 200oer Jahre kam es in vielen Ländern Subsahara-Afrikas zu einem regelrechten JatrophaBoom, von dem riesige Flächen Ackerland betroffen waren und dem in der wissenschaftlichen Debatte um land grabbing ein hohes Maß an Aufmerksamkeit zuteilwurde - nicht zuletzt aufgrund der vielfältigen Konflikte, die er mit sich brachte (Campion/Acheampong 2014; Hunsberger 2010; Schrader 2014; von Maltitz et al. 2014). Mittlerweile ist dieser Boom vorüber und die meisten 
Unternehmen konzentrieren sich auf den Anbau anderer Kulturpflanzen, insbesondere so genannter flex crops wie Mais, Soja, Ölpalmen oder Zuckerrohr, die je nach Nachfrage und Marktpreis flexibel als Nahrungs- oder Futtermittel sowie zur Herstellung von Kosmetika und Agrarkraftstoffen dienen können (Borras et al. 2016). Die Konflikte bestehen jedoch in vielen Fällen weiterhin. Die räumliche Ausbreitung des Jatropha-Anbaus variierte innerhalb Ghanas und betraf vor allem die drei nördlichen Regionen (Northern Region, Upper East, Upper West) sowie Brong-Ahafo und Ashanti ${ }^{2}$ (Yirrah 2012).

Da ich die Annahme vertrete, dass die unterschiedliche Ausgestaltung traditioneller Herrschaftssysteme relevant für das Handeln traditioneller Autoritäten ist, war ein weiteres Kriterium meiner Fallauswahl die Varianz traditioneller Herrschaft innerhalb des gleichen Länderkontexts. In der Ashanti Region im Süden von Ghana sind traditionelle Autoritäten besonders mächtig. Sie sind in stark hierarchischen Strukturen organisiert, mit dem einflussreichen Asantehene, dem König der Ashanti, an der Spitze. Darüber hinaus verfügen sie über sehr gute nationale und internationale Kontakte in Wirtschafts-, Politik- und Gebernetzwerke. In den drei nördlichen Regionen ist die traditionelle Herrschaft fragmentierter als in der Ashanti Region. Ihre Ausgestaltung unterscheidet sich insofern, als dass es in jeder der Regionen mehrere kleinere chiefdoms gibt und nicht ein einziges großes Königreich wie in der Ashanti Region. Die einzelnen kleinen chiefdoms verfügen ebenfalls über eine interne Hierarchie, die vergleichbar mit derjenigen der Ashanti Region ist. Insgesamt stehen ihnen im Verhältnis weniger Ressourcen zur Verfügung, sie sind weniger gut in internationale Netzwerke eingebunden und verwalten insgesamt kleinere Flächen an Land. Hinsichtlich der Organisation des Zugangs zu Land sind die beiden Regionen vergleichbar. Die Projekte ScanFarm und BioFuel Africa wurden relativ zeitgleich von norwegischen Investoren initiiert und die ursprünglich geplante Anbaufläche hatte ein ähnliches Ausmaß. Im Kontext der Landvergabe an die Unternehmen kam es in beiden Fällen zu Konflikten, in denen traditionelle Autoritäten Verhandlungspartner und Repräsentanten der Gemeinschaft sowie Konfliktschlichter waren.

Die empirischen Daten für die Fallstudie habe ich im Verlauf von drei Feldforschungsaufenthalten in Ghana im November 2015, von Februar bis Juni 2016 und von Februar bis März 2017 erhoben. Insgesamt habe ich 46 teilstrukturierte Leitfaden-Interviews in der Hauptstadt Accra, den Regionalhauptstädten der Ashanti und Northern Region Kumasi und Tamale, 
der Hauptstadt des Asante Akyem North Districts Agogo sowie in neun von den agrarindustriellen Projekten betroffenen Dörfern geführt. Interviewpartner*innen waren Mitarbeiter*innen von Behörden auf nationaler, regionaler und Distriktebene, von NGOs (Non-Governmental Organizations) und zivilgesellschaftlichen Organisationen, leitende Angestellte der Unternehmen, Landnutzer*innen der betroffenen Dörfer, traditionelle Autoritäten und ihre Vertreter, Repräsentanten der lokalen Customary Land Secretariats (CLS) (formalisierte traditionelle Landrechtsinstitution) sowie des House of Chiefs in beiden Regionen und auf nationaler Ebene. Zusätzlich zu den Einzelinterviews habe ich sieben Gruppendiskussionen mit insgesamt 176 betroffenen Landnutzer*innen und traditionellen Autoritäten in den beiden Projektgegenden geführt.

\subsection{Struktur des Buches}

Dieses Buch ist wie folgt aufgebaut. Im Anschluss an die Einleitung stelle ich den aktuellen Stand der Forschung zu Konflikten im Kontext von land grabbing und traditionellen Autoritäten in Konflikten um Zugang zu Land dar und zeige relevante Anknüpfungspunkte für meine eigene Forschung auf. Das dritte Kapitel bildet die theoretische Grundlage der Forschung. In diesem entwickle meinen Analyserahmen und führe aus, wie sich dieser für die Analyse der empirischen Daten operationalisieren lässt. Darauf folgend erläutere ich im vierten Kapitel das Design und methodische Vorgehen meiner Forschung. Kapitel fünf dient als Überleitung zur empirischen Analyse und gibt einen Überblick über den Länderkontext Ghanas. Der Fokus dieses Kapitels liegt auf der Entwicklung von Landrechten und traditioneller Herrschaft. Kapitel sechs widmet sich der Darstellung der empirischen Fälle. Im Zentrum stehen der Ablauf der Landvergabe an die Unternehmen ScanFarm und BioFuel Afri$c a$, die im Kontext der Landvergabe auftretenden Konflikten sowie die Konfliktakteure und ihr Handeln. Anschließend analysiere ich in Kapitel sieben, inwiefern sich im Rahmen der großflächigen Vergabe von Land die Kontrolle über den Zugang zu Land verändert, und inwiefern die Veränderung von Kontrollverhältnissen den Zugang unterschiedlicher Landnutzer*innen zu Land beeinflusst. Ich untersuche, welche Akteure vor der Landvergabe Kontrolle über den Zugang zu Land ausgeübt haben, über welche Mechanismen Landnutzer*innen Zugang zu Land erlangt haben und wie dies sich im Rahmen der Landvergabe verändert hat. Aufbauend auf diesen Erkenntnissen arbeite 
ich im achten Kapitel typische Handlungsstrategien heraus, über die traditionelle Autoritäten im Rahmen der Landvergaben Macht ausüben, um den Zugang zu Land zu kontrollieren. Anschließend analysiere ich, inwiefern sich ihre Handlungsstrategien auf die Entstehung und den Verlauf von Konflikten auswirken.

Im Fazit fasse ich die Ergebnisse meiner Analyse zusammen. Ich arbeite Parallelen und Unterschiede zwischen den Fällen heraus und diskutiere, womit diese zusammenhängen. Auf der Grundlage der empirischen Erkenntnisse formuliere ich drei Hypothesen zur Erklärung von Konflikten um Zugang zu Land in traditionellen Landverwaltungssystemen und erörtere die Reichweite dieser Hypothesen. Schließlich zeige ich auf, zu welchen Forschungsfeldern ich mit diesem Buch einen Beitrag leiste. 